\title{
Three-Dimensional Imaging of Point Defects in Functional Materials Using Quantitative STEM
}

\author{
Jinwoo Hwang
}

Department of Materials Science and Engineering, The Ohio State University, Columbus, OH 43212

Many important properties of nanoscale functional and energy-related materials are determined by individual point defects, such as impurity dopant atoms and vacancies. Therefore, to understand the materials physics and exploit the properties of emerging materials, it is crucial to precisely determine the 3D location, distribution, segregation, pairing, and clustering of point defects. Electron microscopy has been previously used to identify individual point defects. For example, annular dark-field (ADF) imaging in STEM is sensitive to the atomic number of the material, and therefore can provide intuitive information on the lateral positions of the impurity atoms [1]. To complete the 3D information, however, the precise depth location (the direction parallel to the electron beam) of the point defects must be uncovered as well. However, acquiring depth information at the atomic scale is challenging. Even with high probe convergence angles, the depth resolution of STEM remains above $\sim 10 \mathrm{~nm}$ [2], which makes it difficult to determine the 3D position of point defects using conventional approaches, such as focal series reconstruction or confocal microscopy. These conventional techniques also require the acquisition of multiple images of the same area of the sample, and therefore may suffer from low precision due to sample drift, or radiation damage due to long beam exposure. The development of a 3D electron microscopy technique that combines resolution, precision, and efficiency is therefore required.

We present a new 3D atomic scale imaging technique based on quantitative STEM. Quantitative STEM measures the ADF signals on an absolute scale, which can be directly compared to simulated images and therefore allows for straightforward interpretation of the image in terms of the number of atoms and atomic species [3]. We show that by including the probe channeling information in the analysis of the atomic column intensities in quantitative STEM images, the 3D structural information of point defects can be determined with unparalleled depth precision [4]. Also, our technique only requires one single ADF image per sample area, and therefore it can achieve substantially higher efficiency in data acquisition and reduce radiation damage to the sample.

For example, we determined the 3D positions of individual $\mathrm{Gd}$ dopant atoms and their nanoscale clustering in $\mathrm{SrTiO}_{3}$. Here, the quantified error (2D Gaussian function in the inset in Fig. 1A) sets practical limit to the dopant visibility and depth precision in the experiment (Fig. 1A). Possible sources contributing to the error include experimental instabilities, such as specimen drift and surface roughness of the sample. Multislice simulations show that the intensities of the atomic columns containing dopants monotonically depend on the number and the depth position of the dopants when the $\mathrm{SrTiO}_{3}$ thickness is $<4 \mathrm{~nm}$ (Fig. 1B). The deviation between the experimental and simulated data points in Fig. 1B is due to the same experimental error shown in Fig. 1A. Therefore we can calculate the distance between each experimental and simulated data points in Fig. 1B, and then weight it with the $2 \mathrm{D}$ error function to acquire the expectation value and uncertainty of the depth position of the dopants [4]. The depth uncertainty of the measurement was less than one $\mathrm{SrTiO}_{3}$ unit cell (u.c.) (Fig. 1D).

We will also discuss recent results and challenges of the comprehensive 3D mapping of individual dopants that control the performance of the novel semiconductor devices. Identifying the 3D 
information of lighter dopants that have atomic numbers close to that of the host atom (e.g. Al or In doped GaN) poses additional challenges, as the intensity in ADF imaging primarily depends on the atomic number. Achieving higher depth resolution and precision will be critical for this work. We advance the 3D quantitative STEM technique in an FEI Titan STEM with a probe aberration corrector that ensures sub-Angstrom lateral resolution, and a high-brightness electron source that provides an excellent signal-to-noise ratio, and therefore allows for substantially higher precision and efficiency in 3D imaging. The new quantitative STEM experiments also utilize the multiple ADF detectors in our STEM. The atomic column image intensities containing depth information varies depending on the collection angle of the ADF detector. Therefore the acquisition of multiple ADF images using different collection angles can provide additional depth information, and lead to a maximized precision for pinpointing the depth of impurity atoms [e.g. 5]. We also expect our new 3D imaging technique to help identify the point defects in other important functional materials, including the dopant atoms in novel quantum devices and the vacancies at oxide interfaces that may govern the properties.

\section{References}

[1] P. M. Voyles, et al., Nature 416 (2002) p. 826.

[2] P. M. Voyles, D. A. Muller, and E. J. Kirkland, Microsc. Microanal. 10 (2004) p. 291.

[3] J. M. LeBeau, S. D. Findlay, L. J. Allen, and S. Stemmer, Phys. Rev. Lett. 100 (2008) p. 206101.

[4] J. Hwang, et al., Phys. Rev. Lett. 111 (2013) p. 266101.

[5] J. Y. Zhang, J. Hwang, B. J. Isaac, and S. Stemmer (submitted).
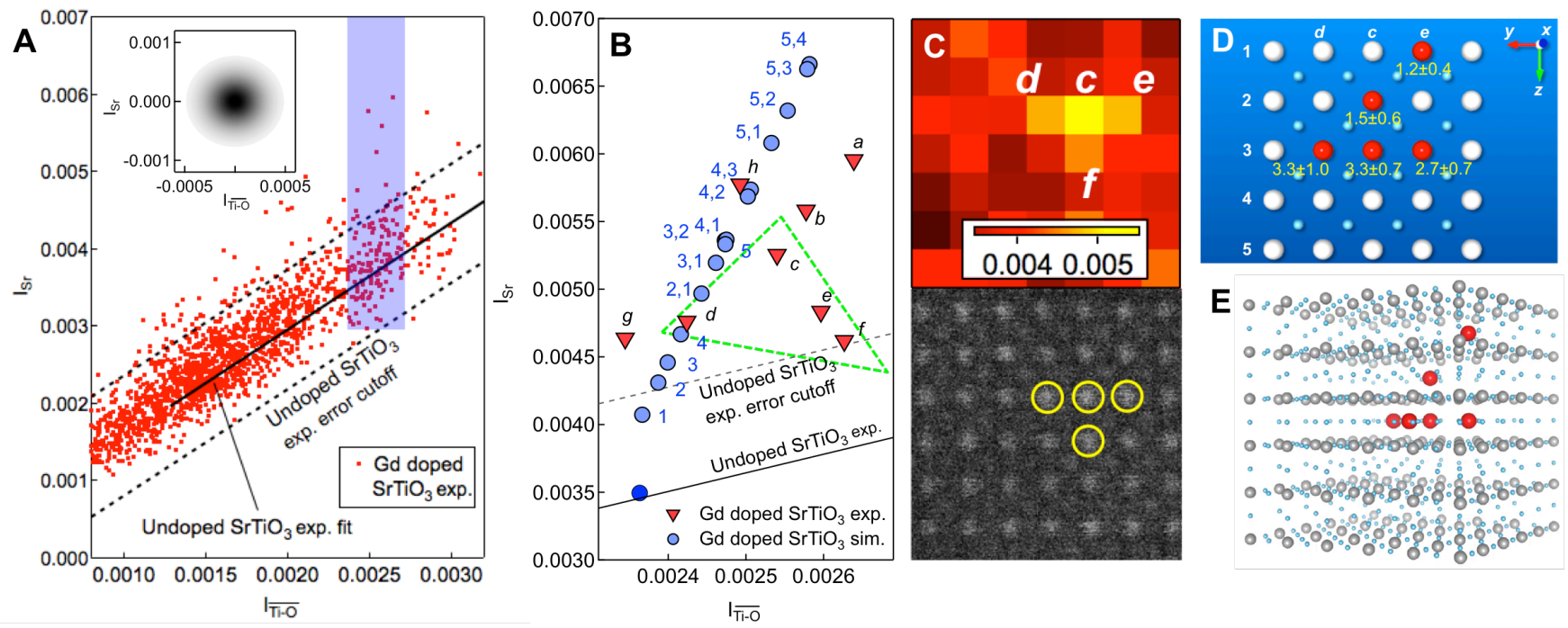

Figure 1. (A) Experimental Sr column intensity $\left(I_{s r}\right)$ vs. mean Ti-O column intensity ( $\left.I_{\overline{T i-O}}\right)$ plot for Gddoped $\mathrm{SrTiO}_{3}$, and fit for undoped $\mathrm{SrTiO}_{3}$ (solid line). The inset shows the 2D error function calculated from the undoped $\mathrm{SrTiO}_{3}$ data. The error cutoff is also shown as dashed lines. The data points above the upper limit of the error cutoff indicate the columns that contain at least one dopant. (B) Multislice simulations (blue circles) of column intensities for the blue rectangular region in Fig. 1A (thickness $=5$ u.c.). The labels (numbers) indicate the dopant position in the column as defined in (D). Experimental points, labeled $a-g$ are also shown. The large dashed triangle indicates the dopant clusters shown in (CE). (C) $I_{s r}$ map (top) and ADF image (bottom) of the area containing $c$ - $f$ columns. (D) A schematic and (E) $3 \mathrm{D}$ rendering of dopant clustering in $c$-e columns. The most probable dopant position is shown in red and the expectation values and uncertainties are labeled (unit = u.c.). 